\title{
Effect of nitric oxide synthase inhibitor on methamphetamine-induced dopaminergic neurotoxicity in rats
}

\author{
Kazue Kikuchi-Utsumi, Masahiko Watabe, Nobuko Matsumura, Toshio Nakaki \\ Department of Pharmacology, Teikyo University, School of Medicine, Japan
}

Methamphetamine (METH) is a psychostimulatory drug with addictive potential. This activity is associated primarily with the stimulation of dopamine release. METH is most harmful to dopaminergic neuron, and its administration to rodents causes long-term damage to dopaminergic terminals in the striatum. We previously demonstrated that BMY7378, $\alpha$ 1Dadrenoceptor antagonist, is completely abolished the METH-induced neurotoxicity without alteration of the striatal extracellular level of dopamine, suggesting an essential role for $\alpha 1 \mathrm{D}$-adrenoceptor to the METH neurotoxicity in rats. A functional interaction between glutamate and nitric oxide (NO) has been implicated in the METH-induced neurotoxicity; however, this issue still remains controversial. This study was designed to investigate on the relationship between the neuroprotective effects of BMY 7378 and the striatal NO and glutamate levels in METH-injected rats.

The rats were intraperitoneally injected with METH $(6 \mathrm{mg} / \mathrm{kg}), 4$ consecutive times at 2-h intervals. Pretreatment with $\alpha 1$-adrenoceptor antagonist (prazosin, BMY7378) or S-methyl-L-thiocitrulline (SMTC), NO synthase inhibitor, was administered intraperitoneally $30 \mathrm{~min}$ before each METH injection. METH-induced hyperthermia, one of the exacerbating factors of this neurotoxicity, was monitored $1 \mathrm{~h}$ after each METH injection with a rectal thermistor probe. Extracellular levels of dopamine, glutamate, and citrulline (an indirect marker for NO synthesis) in the striatum during METH administration was analyzed by in vivo microdialysis. Five days after METH administration, striatal dopamine level was quantified by HPLC.

METH treatments induced hyperthermia and a decrease in the striatal dopamine level. In-vivo microdialysis analysis revealed that METH led to an increase in extracellular citrullin and glutamate levels in the striatum. Pretreatment with prazosin (non-selective $\alpha 1$-adrenoceptor antagonist), or BMY7378 was completely abolished the METH neurotoxicity with a partial reduction of the hyperthermia. These antagonists also strongly prevented increases in extracellular levels of citrulline and glutamate. On the other hand, pretreatment with SMTC did not affect METH-induced hyperthermia. Although this inhibitor exhibited complete inhibition of increases in extracellular glutamate and citrulline levels, the METH-induced neurotoxicity was not ameliorated.

These results suggest that METH-induced NO production is not directly associated with METH-induced dopaminergic neurotoxicity in rat striatum. 